\title{
24 \\ Visual Modeling of Temporal Data in Usability Experiments
}

Sônia Fernandes Silva

Departamento de Engenharia Elétrica, Universidade Federal da Paraiba C.P.10106 Campina Grande PB, Brasil, Tel/Fax: +55-83-3101015, sonia@dsc.ufpb.br / silva@dis.uniroma1.it

Tiziana Catarci

Dipartimento di Informatica e Sistemística, Università di Roma "La Sapienza"

Via Salaria, 113 I-00198, Rome, Italy, Tel/Fax: +39-6-49918331, catarci@infokit.dis.uniroma1.it / tic@cs.brown.edu

\begin{abstract}
In most recent Visual Query Systems (VQSs), there is the attempt to address the different needs of various classes of users by developing adaptive interfaces having a fundamental component in the user model module. Within this context, we argue that the temporal issues are a key factor in modeling th user's interaction, and also in carrying out related usability analysis on VQSs. Hence, we propose a uniform framework for modeling and manipulating several kinds of time-based information related with VQSs, which exploits a graph-based formalism, equipped with visual temporal operators. In this paper, we concentrate on the time-based information which are derived from usability experiments. In particular, we show that the history of the user's interaction can be accessed and dynamically maintained by using such visual temporal operators. This approach is an effort towards clearly establishing a link between user interaction and modeling and data modeling and querying, allowing a structured and well controlled analysis of VQS usability results.
\end{abstract}

\section{Keywords}

Visual Query Systems, User Model, Usability Experiments, Temporal Data

\section{INTRODUCTION}

As a consequence of the user-centered approach to the development of information systems, several research activities on easy-to-use visual systems have been carried out in the past years. Among the systems specifically devoted to the extraction of information from databases, there are the Visual Query Systems - VQSs (see (Catarci et al. 1997) for a survey).

*Partially supported by Brazilian CNPq. 
In most recent VQSs, there is the attempt to address the different needs of disparate classes of users by developing interfaces which automatically adapts to such users, based on a user model (a user model is a system knowledge source that contains explicit assumptions on all aspects of the user that may be relevant for the dialogue behavior of the system (Kass \& Finin 1989)). The system adequacy is then checked by running suitable usability experiments involving samples of representative users. Most of such experiments are comparative studies among the different ways to access information, namely, textual interaction, natural language and visual interaction (Resneir 1988), (Bell 1993), (Shneiderman 1994). Recently, other experiments have been performed, in order to explore the effectiveness of VQSs providing various kinds of users with different visual representations and access methods. The experimental results indicate that the appropriateness of a visual representation is strictly related with the level of users'skill and the kind of query (Leventhal et al. 1995), (Paton et al. 1995), (Badre, Catarci \& Santucci 1996), (Catarci et al. 1996).

We propose a uniform framework suitable in principle to model different kinds of temporal information related with VQSs, namely those contained in historical databases as part of the application data (e.g. the employees'salary history); those contributing to the definition of the user model (e.g. the history of the user's accesses to the database); and those which are part of the data set coming out from usability experiments (e.g. the completion times of the users'queries). The overall framework is built on top of a graph-based formalism, equipped with visual temporal operators (preliminary results on the visual temporal operators have been presented in (Fernandes Silva, Schiel \& Catarci 1997)). Such a formalism is an extension of the Graph Model (Catarci, Santucci \& Angelaccio 1993), so as to model temporal issues.

In this paper, we concentrate on the last application of the framework. In particular, we first show that the history of the user's interaction can be accessed and dynamically maintained by applying the visual temporal operators, as an effort of clearly establishing a link between user interaction and modeling and data modeling and querying. Then, we model in our framework the data which come from the usability experiments and use the visual temporal operators to access such data in order to perform a structured and well controlled analysis of the VQS ease-of-use.

The paper is organized as follows. Section 2 summarizes the temporal extension of the Graph Model and describes the user model component, formalizing its temporal aspects. Section 3 introduces the visual temporal operators and show their usage in visually expressing temporal queries. Section 4 reports some usability experiments on VQSs, and shows how the temporal data used in such experiments are modeled using our formalism. Section 5 draws the conclusions. 


\section{THE TEMPORAL GRAPH MODEL}

In this section we present the temporal extension of an object-based model, the Graph Model, originally introduced in (Catarci et al. 1993), for visually representing and querying databases.

A Graph Model Database (GMDB) is a triple $\langle g, c, m\rangle$, where $g$ is a Typed Graph, $c$ is a set of Integrity Constraints and $m$ is an Interpretation. The schema of a database, i.e. its intensional part, is represented in the Graph Model by the Typed Graph and the set of Constraints. The instances of a database, i.e., its extensional part, are represented by the notion of Interpretation. A database schema is expressed in the Typed Graph in terms of classes and relationships among classes (called roles). A class is an abstraction of a set of objects with common characteristics. The relationships among classes represent associations among objects of the classes.

In the temporal extension of the Graph Model, classes and relationships can also be modeled as temporal components of temporal databases, so allowing the user to retrieve data not only concerning the current state of the database, but also its past states (historical records). In particular, in this work we deal with historical databases, where each instance is associated with a set (called lifespan) of disjoint intervals of values of a linearly ordered and discrete time domain. Each interval is represented by an ordered pair of natural numbers. In particular, we assume that every lifespan is normalized, which means that no pairs of intervals in the lifespan meet (an interval $\mathrm{A}=\left\langle x_{1}, x_{2}\right\rangle$ meets an interval $\mathrm{B}=\left\langle x_{3}, x_{4}\right\rangle$ if $x_{2}=x_{3}$ or $\left.x_{1}=x_{4}\right)$. In short, we share with the majority of temporal data models the notion that the time domain is linearly ordered, discrete, bounded in the past and unbounded in the future (Morris \& Khatib 1997).

An example of using the Graph Model, in order to model the information concerning employment agencies is presented in Figure 1 and Table 1. Figure 1 shows the typed graph $g$ with the constraints. Table 1 contains a possible interpretation $m$ for $g$. For the sake of simplicity, the interpretation is listed only for a subset of the nodes.

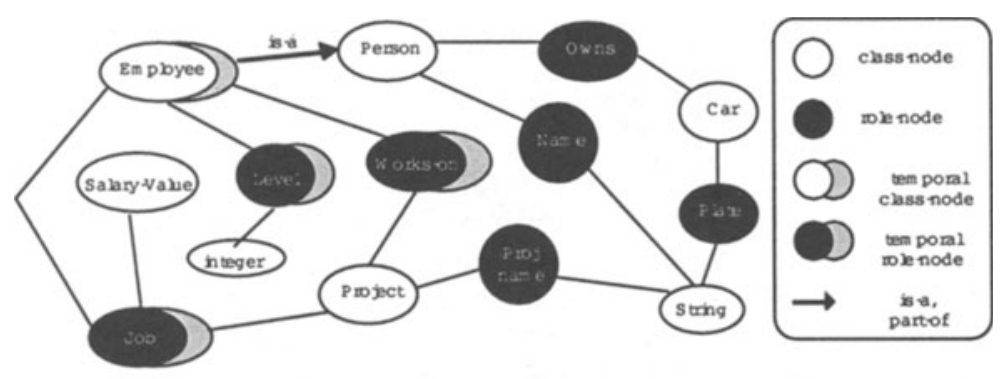

Figure 1 A Typed Graph $g$ and a Set of Constraints $c$ 


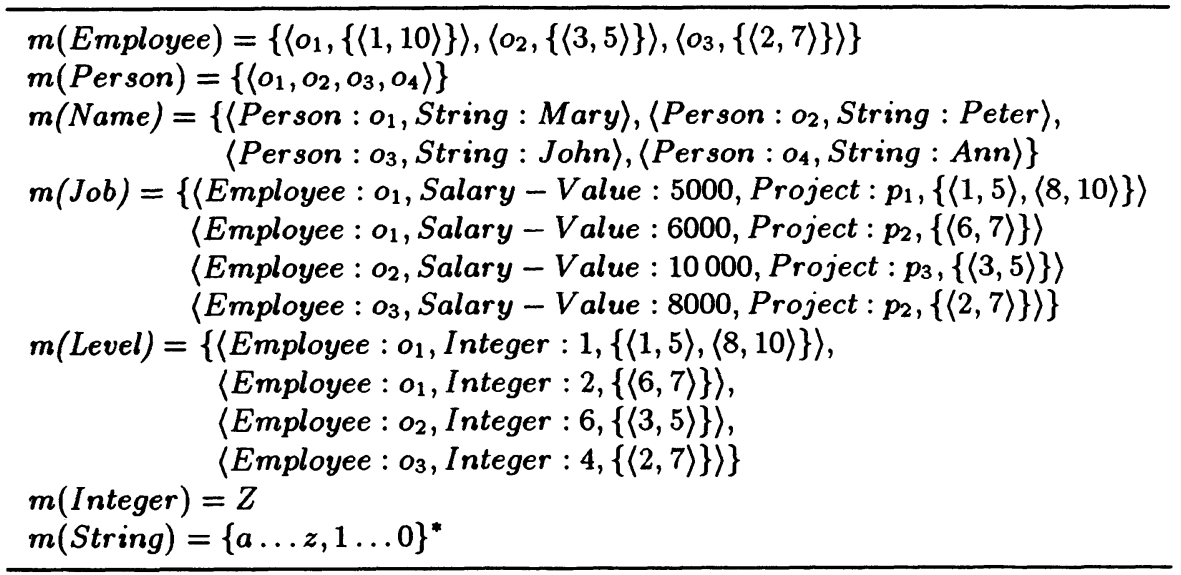

Table 1 An Interpretation $m$ for $g$

The details of the Graph Model and its temporal extension is out of the scope of this paper. The interested reader can refer to (Catarci et al. 1993), (Catarci, Santucci \& Cardiff 1997), (Fernandes Silva et al. 1997).

\subsection{The Temporal User Model}

\section{(a) Basic Notions}

We have adopted the user model defined in (Catarci et al. 1996), which automatically provides the user with the most appropriate visual representation (form-based, iconic, diagrammatic and hybrid) and the corresponding interaction modality, according to his or her skill and needs. Such a user model consists of three components: the class stereotype (CS), the user signature (US) and the system model (SM). The class stereotype denotes the user's profile, the user signature corresponds to the history of user's interactions and the system model is the user's view of the system application.

Concerning the class stereotype, our user classification scheme is the one proposed in (Chang, Costabile \& Levialdi 1993), where the database users are professional or non-professional ones, depending on the training they have had. Concerning only the non-professional users, four user features related with the querying task are considered: frequency of interaction, repetitiveness of the query, structural complexity of the query, familiarity with the database content (see (Chang et al. 1993), (Catarci et al. 1996)). For each one of such features, two extreme values High $(H)$ and Low $(L)$ are considered. So, sixteen user classes are generated, ranging from the class "ex temporary-occasionalnaive-unfamiliar", whose feature values are all $\mathrm{L}$, to the class "repetitivefrequent-sophisticated-familiar", whose feature values are all H. Among the 
possible sixteen classes, four are unlikely, they are the classes whose value of the feature frequency of interaction is low, while the value of the feature familiarity with the database content is high. Since the same type of visual representation is the most appropriate for some of the twelve user classes, they were reduced to five stereotypes (see (Catarci et al. 1996) for more details).

Concerning the user signature, the system keeps track of the set of queries the user formulates during her/his interaction with a database. At any moment, the values $\mathrm{L}$ and $\mathrm{H}$ of each feature can change. As a consequence, the stereotype also changes. In order to model this dynamic behavior, the framework of Chang et al. (Chang et al. 1993) proposes a state-transition diagram, whose nodes are the twelve user classes and the edges represent changes of the values from low to high and vice-versa.

The third component of the user model, the system model, is the representation of the knowledge the user has of the content of the database and its structure, that is, the user's database view (Catarci et al. 1996). In this case, the system constructs a historical of views that must be analyzed from time to time in order to suggest the user the database view most appropriate for his or her needs.

\section{(b) Structural Aspects}

As we said above, the user model for an adaptive VQS is a triple $U M=$ $\langle C S, U S, S M\rangle$, where $C S$ is the user class stereotype, $U S$ is the user signature and $S M$ is the system model. For a dynamic update of the $C S$ component, the user features are computed starting from the $U S$ component, which contains all the history of interaction of each user. An interaction is represented by a temporal sequence of queries $Q=\left\langle Q_{1}, Q_{2}, \ldots, Q_{n}\right\rangle$ formulated at time $t_{1}, t_{2}, \ldots, t_{n}$ respectively. More precisely, the user's interaction with the system can be specified in terms of three components: the Session, which identifies each specific interaction that happens during a certain time interval; the Query, which contains the specification of each query performed during a certain session; the Modality, which, for each query, signals the set of modalities (form-based, diagrammatic, iconic, etc) used for formulating it. In other words, during an interaction the user performs a temporal sequence of queries, specified by one or more modalities (each one within a time interval). The status of each query can be Completed or Interrupted, indicating that the query has been successfully completed or not.

Given its temporal characteristics, we can represent the $U S$ component of the user model as a particular GMDB, which is called Interaction GMDB. Figure 2 illustrates the corresponding Typed Graph.

The class-node STEREOTYPE is an aggregation (the aggregation concept is usually defined in semantic and conceptual data models (Hull \& King 1987), and is represented as a part-of constraint in the Graph Model) of the classnodes FREQUENCY, REPETITIVENESS, COMPLEXITY and FAMILIAR$I T Y$ (whose instances are the binary values $\mathrm{L}$ or $\mathrm{H}$ ), where there is a role- 


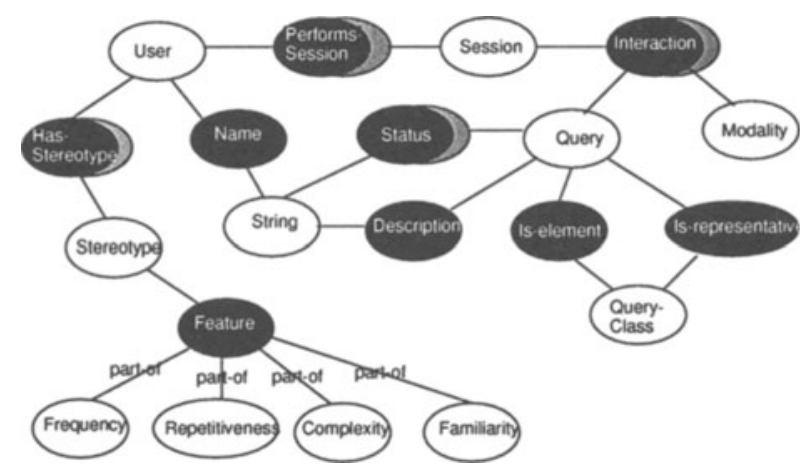

Figure 2 Typed Graph of Interaction $G M D B$

node FEATURE between the aggregate class and the component classes. In this case, a "repetitive-occasional-sophisticated-unfamiliar" user is represented by an identifier in the class USER, for instance $o_{1}$, the tuple $\langle$ User : $o_{1}$, Stereotype : $\left.s_{1}\right\rangle$ in the temporal role-node HAS-STEREOTYPE and the tuple 〈Stereotype : $s_{1}$, Repetitiveness : H, Frequency : L,Complexity : $H$, Familiarity $: L\rangle$ as instance of the role-node FEATURE. The class-node QUERY is associated to the class-node QUERY-CLASS through the rolenodes IS-ELEMENT and IS-REPRESENTATIVE. It means that, according to (Catarci et al. 1996), a set of queries $\left\{Q_{1}, Q_{2}, \ldots, Q_{n}\right\}$ having similar patterns are grouped into query classes $\left\{C_{1}, C_{2}, \ldots, C_{m}\right\}$, with $m \leq n$, where each query class $C_{k}$ has a representative query (see the procedure defined in (Catarci et al. 1996), which identifies whether a query belongs to a particular query class). The temporal role-node STATUS represents the status history of each specific query the user formulates.

The interpretation of the temporal role-node INTERACTION is made of labeled tuples, associating objects from SESSION, QUERY and MODALITY to a lifespan $l s=\left\{I_{1}, \ldots, I_{n}\right\}$, comprising the intervals during which a certain modality is used (within specific query and session). For instance, if the initial state of a user interaction with a database is the triple $\left\langle S_{0}, Q_{0}, M_{0}\right\rangle$ at time $t_{0}$, and the user changes the modality $M_{0}$ into $M_{1}$ at time $t_{1}$, a tuple $\left\langle\right.$ Session : $S_{0}$, Query : $Q_{0}$, Modality : $\left.M_{0},\left\langle t_{0}, t_{1}\right\rangle\right\rangle$ is added to the interpretation of INTERACTION.

\section{TEMPORAL VISUAL QUERY OPERATORS}

In (Catarci et al. 1993) it is demonstrated that any query-oriented user interaction with a database can be expressed in terms of a simple set of fundamental Graphical Primitives - GPs. In particular, two primitives are presented: selection of a node and drawing of an edge, and it is proved that all first order queries can be expressed by composing the two primitives. 
In order to express temporal queries, we have extended the GP set with other visual primitives, namely temporal visual query operators (TVQOs). The TVQOs are applied to both temporal classes and temporal relationships as components of a GMDB. They are called snapshot and slice for temporal selection, and time-display for temporal projection. Temporal selection is a logical condition, based on a predicate that involves the time associated with the facts, while temporal projection returns the time values associated with the data derived from temporal selection (see (Jensen et al. 1994)).

We use the snapshot operator for the extraction of an instantaneous fact (valid at a single time-instant) and the slice operator for the extraction of a historical fact (with a longer lifetime). The time-display operator is used to display time intervals, instants and/or durations in different granularities in the query result. It is also used in queries that involve the combination of the snapshot and slice operators, more specifically, in queries with a temporal reference to another data. For instance, in order to evaluate the query "Which salaries did the employees earn when they changed their level for the first time?", the slice operator on the temporal relationship LEVEL is used for requesting the time when the level changed, and the snapshot operator for retrieving the corresponding salary, in this case, we use the time-display operator on the role-node resulting from the slice operator on the temporal relationship $L E V E L$, with parameter first - interval.

In the following, we summarize an adaptive VQS for historical databases (Fernandes Silva \& Catarci 1998), called TVQE (Temporal Visual Query Environment), on which a temporal query can be visually expressed by using the TVQOs.

According to (Catarci et al. 1993), a typical structure of the query specification process is constituted by three interaction phases: Location, Manipulation and Visualization. The TVQE interface is composed by three windows: Schema Window, Temporal Window and Data Visualization Window, each one represents a query phase. Moreover, TVQE supports three visual representations of the database schema, namely, form-based, diagrammatic and iconic representations. The user may change the visual representation at any moment, by selecting the button corresponding to the new one, and all operations performed up to that point are automatically restored according to the new representation.

\subsection{Selection of a Node}

Let $D=\langle g, c, m\rangle$ be a $G M D B$. The user's clicking on a node corresponds to the GP selection of a node, through which the state of a node is switched from the value unselected to either the value selected, which means included in the schema of interest, or displayed, which means included in the query result (see (Catarci et al. 1993) for more details). Figure 3 shows the schema 
window, which represents the location phase. The visual mechanism used in this phase is the GP selection of a node.

Assuming that the user is interested in knowing "Which salaries did the employees earn when they changed their level for the last time", s/he first accesses a database schema. As a consequence, the unprintable classes which compose the selected schema appears in the list box, and one of them is selected as the trigger class of the query (Kim 1989). Next, the schema, rooted at the trigger class, is visually represented. In our example, EMPLOYEE is the selected trigger class, and the form-based representation is displayed in Figure 3 (the system provides the user with the most appropriate visual representation, based on the user model suggestions).

The user can change the status of a class or relationship by selecting the radio button corresponding the status, as shown in Figure 3. In our example, EMPLOYEE and LEVEL are the selected classes, while SALARY and NAME are displayed. Next, the system generates the appropriate sub-schema. The user can either save the corresponding sub-schema for further manipulation or immediately use it in the manipulation phase. Note that the temporal components are visually distinguished in any representation.

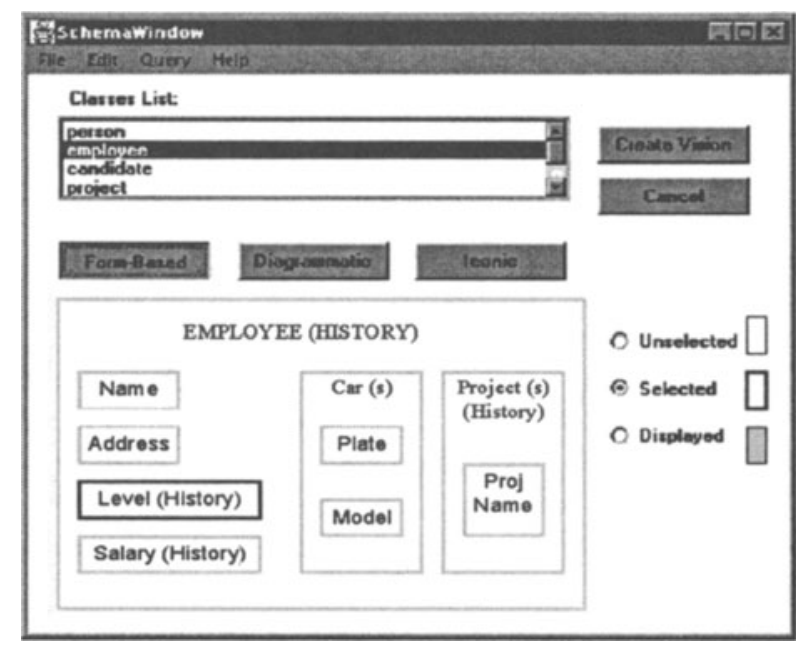

Figure 3 Form-Based Representation of a Database Schema

\subsection{Snapshot and Slice Operators}

Let $D^{\prime}=\left\langle g^{\prime}, c^{\prime}, m^{\prime}\right\rangle$ be the $G M D B$ resulting from the node selections. The application of a TVQO on $D^{\prime}$ results in a new GMDB $D^{r}=\left\langle g^{r}, c^{r}, m^{r}\right\rangle$. The 
next paragraphs will explore the derivation of $D^{r}$ (which is formally defined in (Fernandes Silva \& Catarci 1998)).

The snapshot operator may be applied to temporal class- or role-nodes, with a temporal predicate $p(t)$ of the form begin $(t)$, end $(t)$ and at $(t)$, where $t$ is either a time instant or a time reference to another data. When the snapshot is applied to a temporal class or role-node, a new non-temporal node is created whose interpretation is the set of instances of the original class that satisfy $p(t)$.

The slice operator may be applied to temporal class- or role-nodes, specifying either a temporal predicate $p(I)$, representing the predefined temporal comparison operators among time intervals, defined by Allen (Allen 1983), where $I$ is a time interval, or the operator all, used for retrieving all histories of a temporal class or relationship. When the slice is applied to a temporal class-node $n$, this is transformed into a non-temporal class-node $n^{\prime}$ containing all original instances without lifespans. A new class-node $s$ is created as an aggregation of the class $n^{\prime}$ with the class of time intervals $i$. The same procedure is used when a slice is applied to a temporal role-node $n$. The only difference is that the classes taking part to the aggregation are the classes $n_{1}, \ldots, n_{k}$ originally connected by $n$.

For example, concerning the slice on a temporal role-node, let us consider the temporal relationship JOB among the classes EMPLOYEE, PROJECT and SALARY-VALUE (see Figure 1) and its correspondent interpretation in Table 1. When the slice (with parameter all, for example) is applied on the temporal role-node $J O B$, a new role-node $r$ is created whose related classes are JOB All, EMPLOYEE, PROJECT, SALARY-VALUE and the class ALL (according to the given parameter) representing the time intervals. So, the tabular representation of the interpretation for the role-node $r$ is shown in Table 2.

\begin{tabular}{ccccc}
\hline JOB All & EMPLOYEE & PROJECT & SALARY-VALUE & All \\
\hline$J_{1}$ & $O_{1}$ & $P_{1}$ & 5000 & $\{\langle 1,5\rangle\}$ \\
$J_{2}$ & $O_{1}$ & $P_{1}$ & 5000 & $\{\langle 8,10\rangle\}$ \\
$J_{3}$ & $O_{1}$ & $P_{2}$ & 6000 & $\{\langle 6,7\rangle\}$ \\
\hline$J_{4}$ & $O_{2}$ & $P_{3}$ & 10000 & $\{\langle 3,5\rangle\}$ \\
$J_{5}$ & $O_{3}$ & $P_{2}$ & 8000 & $\{\langle 2,7\rangle\}$ \\
\hline
\end{tabular}

Table 2 Tabular Representation of the Interpretation of the Role-Node $r$

The TVQOs application is performed in the manipulation phase through the temporal window. The temporal window is basically composed by a workspace on which a sub-schema of interest is visually represented and the following buttons: 
- Where button, which represents the data selection, whose visual mechanism is the GP drawing of an edge;

- When button, which represents the temporal selection, whose visual mechanism are the snapshot, slice and time-display operators (the last one is used in queries with temporal reference to another data);

- Data visualization button, which represents the data projection on the nodes with status displayed;

- Time visualization button, which represents the time projection, whose visual mechanism is the time-display operator.

In our example, the user selects the when button applied to the temporal relationship LEVEL. Next, s/he chooses the period radio button (corresponding to the slice operator) and the month granularity in the menu of time granularities, as shown in Figure 4. Then, a dialog box with the now, instant, temporal reference, period and all history options appears. (now and instant options are disabled because they cannot be used in a slice query). The user selects the all history option.

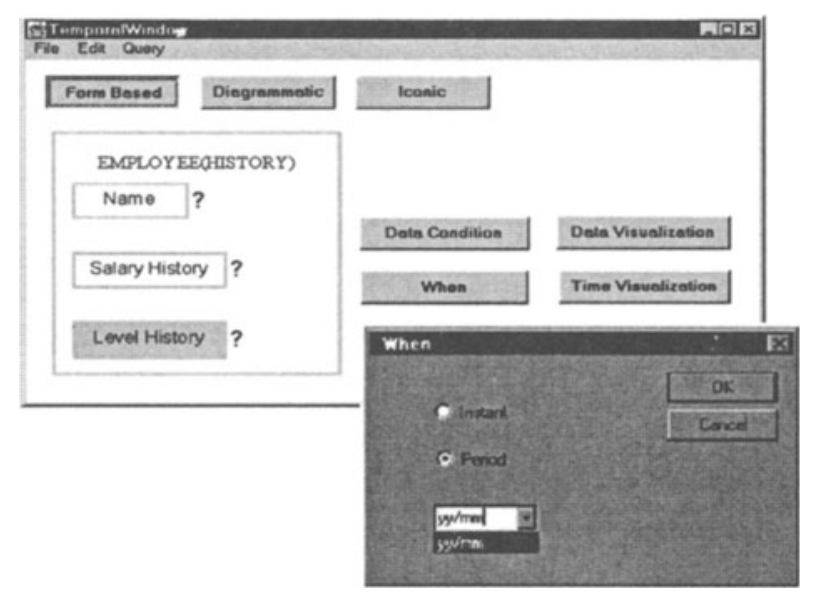

Figure 4 Await Condition of a Slice Query

As we said before, when the slice (with parameter all) is applied on a temporal role-node, $L E V E L$ in the example, a new role-node $r$ is created, whose related classes are in this case LEVEL All, EMPLOYEE, INTEGER, and the class $A L L$ (according to the given parameter) representing the time intervals. 


\subsection{Time-Display Operator}

The time-display operator is used for furtherly manipulate the result of a slice operation, in order to retrieve the lifespan of the various classes. Thus, the time-display is always applied on a role-node $r$, whose related classes are either $s, n^{\prime}$ and $i$ (slice on a temporal class) or $s, n_{1}, \ldots, n_{k}$ and $i$ (slice on a temporal role-node).

The time-display operator is specialized into two operators: interval timedisplay and grouping time-display (the last operator will be defined in Section 4). Through the interval time-display operator, the user can select one or more time intervals from a lifespan, by specifying an ordinal function $\operatorname{ord}(I, p)$, where $I$ is a lifespan associated with the instances of a temporal class or relationship and $p$ is an ordinal number of the form 1st-interval, 2nd-interval, nth-interval or last-interval. By applying the time-display using this function, the tuples in $r$ sharing the same values when projecting them on the class $n^{\prime}$ or on the classes $n_{1}, \ldots, n_{k}$ are time ordered. For example, in Table 2 , the tuples $\left\langle J_{1}, O_{1}, P_{1}, 5000,\{1,5\}\right\rangle$ and $\left.\left\langle J_{2}, O_{1}, P_{1}, 5000,\{8,10\}\right\rangle\right\rangle$ are time ordered, the last lifespan corresponds to the interval $\langle 8,10\rangle)$.

We also use the interval time-display operator in queries that involve the combination of the snapshot and slice operators. The visual counterpart of the time-display, in the TVQE interface, is the time visualization button. By clicking on such a button, the objects which are instances of a temporal class or relationship are visually represented in the data visualization window. Coming back to our example, as a consequence of the slice operation on $L E V E L$, the label "all history" appears beside the temporal relationship $L E V E L$ (this also happens in a data condition, where a predicate that represents a data condition appears beside the selected class), as shown in Figure 5 (where the chosen representation has been changed into diagrammatic). The user can see the history of levels of each employee in the data visualization window, by selecting the time visualization button.

Figure 5 also shows the effects of the user's selection of the when button applied to the temporal relationship SALARY. The same dialog box appears (with the period and all history options disabled). In the example, the user selects the temporal reference option and the LEVEL option in the menu of temporal classes which can be used as temporal references, as shown in Figure 6. Since the history of levels of each employee may contain several time intervals, the user can select one or more of them. So, after s/he has chosen the temporal reference, another dialog box appears to let her/him selecting the first, nth-interval or last interval. In the example, the last interval has been chosen, as shown in Figure 6. At this point, the temporal condition has been completely specified.

Moreover, it is also possible to retrieve the duration of the selected intervals, using the time-display operator. Details about the formalization of the timedisplay, snapshot and slice operators can be seen in (Fernandes Silva \& Catarci 


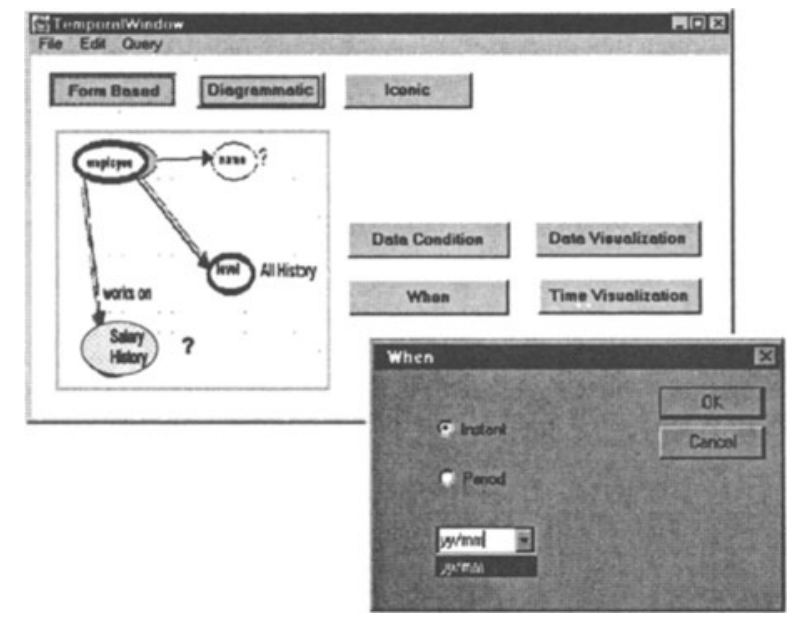

Figure 5 Example of Condition Formulation for a Slice Query

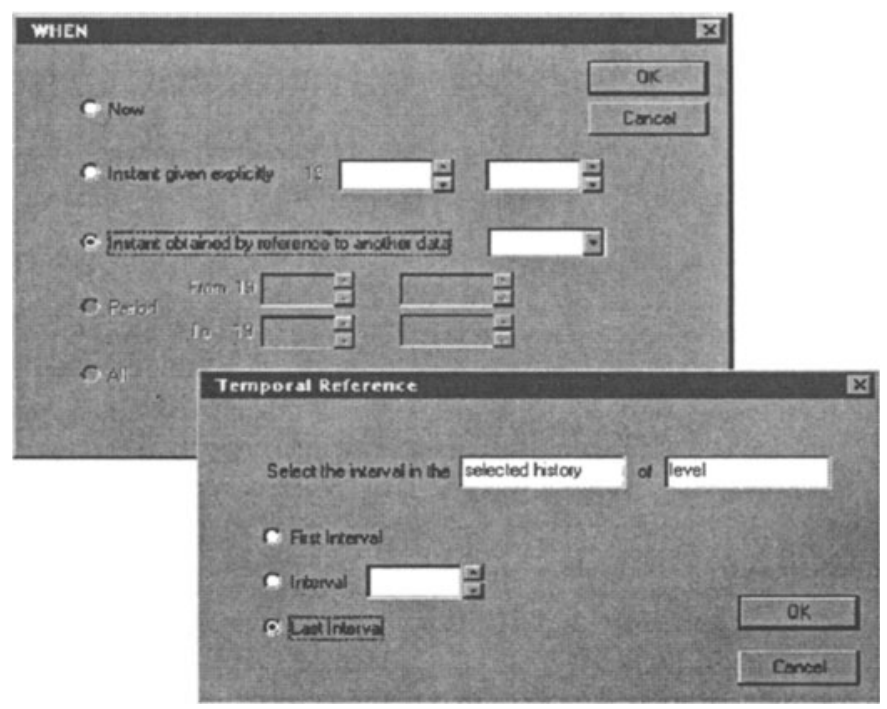

Figure 6 Await Condition of a Snapshot Query with Temporal Reference to Another Data

1998). A preliminary description of the snapshot and slice formalization, for binary relationships only, can be found in (Fernandes Silva et al. 1997). 


\section{USABILITY EXPERIMENTS}

In this section we show how to model in our formalism the temporal issues which are relevant for the usability experiments and how the TVQOs can be used to access such data in order to evaluate the ease-of-use of a VQS. In particular, we concentrate on two different experiments: the first one that evaluates the use of a hypertext system in three museums (Shneiderman et al. 1989), while the second one performs a comparative analysis of the easeof-use of two VQSs with different interaction modalities (diagrammatic vs. iconic) (Badre et al. 1996).

\subsection{Evaluating a Hypertext System}

The experiment reported in (Shneiderman et al. 1989) explores the use of the hypertext system Hyperties, an interactive encyclopedia system, in three museums settings. This experiment is aiming at studying whether the users exploit the hypertext feature (moving from one article to the next) as opposed to using the index access.

In (Shneiderman et al. 1989), the evaluation of Hyperties is divided into two parts. In the first part, a common database was used in two museums and quantitative data were logged by the computer for further experiment analysis. The database consists of articles about a particular subject and, within each article, specific words represent links to another articles. In the second part, a different database is used for a third museum, with direct observation of users and log data. In this paper, we only consider the experiment data used in the first part.

The hypertext system automatically records the time at which each session begins, the name of each accessed article, the number of seconds spent when accessing each article and index. In short, the system recorded data from 373 sessions at B'nai B'rith Klutznick Museum (BBK) and 361 sessions at the International Center of Photography (ICP).

According to the experiment, we have two kinds of users: BBK users (visitors at BBK) and ICP users (visitors at ICP). All users are considered unskilled ones. Since the system uses the hypertext feature, we consider the articles accesses as instances of the class QUERY in the Interaction GMDB (see Figure 2). Each user performs a session $S_{i}$ with a variable number of queries (accessed articles), where each query is expressed by two kinds of modalities, namely $M_{1}$ (representing the index access) and $M_{2}$ (representing the hypertext access) and the system allows the change of modality during the access to a specific article.

In (Shneiderman et al. 1989), the experiment returned the following data: 
- Articles per Session: the number of articles accessed during one session.

- Index accesses per session: the number of times the index was accessed during one session.

- Articles read per index access: average number of articles read per session divided by average number of index accesses per session.

- Duration of session: the sum of the time spent in each article for which a valid time value had been recorded (for the sessions that were abandoned, the time for the last article was not included).

- Percentage of abandoned sessions : the proportion of sessions not terminated by a QUIT command.

- Average time per article: the sum of all recorded time values divided by the number of articles used to obtain the time values.

- Corrected Duration: the duration of a session plus the average time per article for each article whose time value was not included in the duration.

For each of the above items, a correspondent histogram showing the percentage of sessions for each museum was created.

\section{(a) Classes at Temporal Levels}

Before showing how the TVQOs can be used to access the experiment data (which are modeled through the Interaction GMDB), we recall that there is a "temporal dependency" among the components SESSION, QUERY and MODALITY, that is, the union of lifespans of the modalities which are used for formulating a query determines the lifespan of such a query (the same holds between query and session). Within this context, we introduce the concept of classes at temporal levels.

The classes $n_{1}, \ldots, n_{k}$ are at temporal levels if they are connected by a temporal relationship $n$ and a temporal level $l$ is associated to each class $n_{i}$, with $1 \leq l \leq k$, where a class $n_{i}$ at bottom level represents the simplest type of temporal element, which is associated with a single time interval. As a consequence, it follows that:

- The temporal elements which compose the lifespan of a temporal relationship always represent the time domain associated with the related class at bottom level.

- Starting from the class at bottom level, we can derive the lifespan of the class at a certain level $l$ making the union of the lifespans of the class at level $l-1$.

For instance, considering the temporal role-node INTERACTION, the classes MODALITY, QUERY and SESSION can be given the levels 1, 2, 3 respectively, where the level of MODALITY is the bottom level. Thus, we can derive the lifespan of any query (session) by identifying the lifespans of the associ- 
ated modalities (queries). We can do this by using the grouping time-display operator.

Through the grouping time-display operator we can also gather the instances of some classes from $n_{1}, \ldots, n_{k}$. The basic strategy is to exclude a class $n_{l}$ from the set of classes $n_{1}, \ldots, n_{k}$ which are related by the role-node $r$, in order to retrieve the union of time intervals associated with the tuples in $r$ sharing the same values when projecting them on the classes $n_{1}, \ldots, n_{l-1}, n_{l+1}, \ldots, n_{k}$.

So, using the strategy of grouping time-display operator, we see that the lifespan of a class at a certain level $l$ is automatically derived through the recursive application of the grouping time-display with parameters $1, \ldots, l-1$.

For instance, let us consider the data in Table 3 as a sample tabular representation of the interpretation of INTERACTION, with data referring to two different users. In order to retrieve the lifespan of each query in a session, we apply the slice operator on the temporal role-node INTERACTION. Next, we apply the grouping time-display operator on the role-node $r$ whose parameter (the collapsed class) is the class MODALITY. The result is shown in Table 4. In order to retrieve the lifespan of each session, we apply again the grouping time-display with parameter QUERY.

\begin{tabular}{cccc}
\hline SESSION & QUERY & MODALITY & Interval \\
\hline$S_{1}$ & $Q_{1}$ & $M_{2}$ & $\{\langle 0,2\rangle,\langle 5,6\rangle\}$ \\
$S_{1}$ & $Q_{1}$ & $M_{1}$ & $\{\langle 3,4\rangle\}$ \\
$S_{1}$ & $Q_{2}$ & $M_{2}$ & $\{\langle 7,9\rangle\}$ \\
$S_{1}$ & $Q_{2}$ & $M_{3}$ & $\{\langle 10,13\rangle\}$ \\
$S_{1}$ & $Q_{2}$ & $M_{1}$ & $\{\langle 14,15\rangle,\langle 22,25\rangle\}$ \\
$S_{1}$ & $Q_{2}$ & $M_{2}$ & $\{\langle 16,17\rangle,\langle 20,21\rangle\}$ \\
$S_{1}$ & $Q_{3}$ & $M_{1}$ & $\{\langle 26,28\rangle,\langle 33,35\rangle\}$ \\
$S_{1}$ & $Q_{3}$ & $M_{2}$ & $\{\langle 29,32\rangle\}$ \\
$S_{1}$ & $Q_{3}$ & $M_{3}$ & $\{\langle 36,40\rangle\}$ \\
\hline$S_{2}$ & $Q_{1}$ & $M_{2}$ & $\{\langle 1,7\rangle\}$ \\
$S_{2}$ & $Q_{2}$ & $M_{3}$ & $\{\langle 8,9\rangle\}$ \\
$S_{2}$ & $Q_{2}$ & $M_{1}$ & $\{\langle 10,14\rangle\}$ \\
$S_{2}$ & $Q_{2}$ & $M_{3}$ & $\{\langle 15,16\rangle\}$ \\
$S_{2}$ & $Q_{3}$ & $M_{3}$ & $\{\langle 17,20\rangle\}$ \\
$S_{2}$ & $Q_{3}$ & $M_{2}$ & $\{\langle 21,26\rangle\}$ \\
\hline
\end{tabular}

Table 3 Tabular Representation of the INTERACTION Interpretation with Data from Two Different Users

In order to obtain the summary data of the experiment listed above, we apply the TVQOs on the Interaction GMDB. The following operation is a common prerequisite for all cases: 


\begin{tabular}{ccc}
\hline SESSION & QUERY & Interval \\
\hline$S_{1}$ & $Q_{1}$ & $\{\langle 0,6\rangle\}$ \\
$S_{1}$ & $Q_{2}$ & $\{\langle 7,17\rangle,\langle 20,25\rangle\}$ \\
$S_{1}$ & $Q_{3}$ & $\{\langle 26,40\rangle\}$ \\
\hline$S_{2}$ & $Q_{1}$ & $\{\langle 1,7\rangle\}$ \\
$S_{2}$ & $Q_{2}$ & $\{\langle 8,16\rangle\}$ \\
$S_{2}$ & $Q_{3}$ & $\{\langle 17,26\rangle\}$ \\
\hline
\end{tabular}

Table 4 Lifespan of each Query Obtained by Collapsing the Class MODALITY

- Selection of either BBK or ICP users:

The label of the edge $\langle N A M E, S T R I N G\rangle$ is changed into either "BBK" or "ICP".

Then, the experiment data are specified as follows (for the sake of brevity, we just show two examples, the other data may be analogously derived).

\section{1) Articles per Session:}

- The classes USER, PERFORMS-SESSION, SESSION, INTERACTION, QUERY and MODALITY are selected.

- the slice operator is applied on the temporal role-node PERFORMS-SESSION with parameter all.

- The interval time-display operator is applied on the role-node $r$ (resulting from the slice above) without parameters.

- The slice operator is applied on the temporal role-node INTERACTION with temporal predicate 'during(i)'.

- The grouping time-display operator is applied on the role-node $r$ (resulting from the slice above) with parameter MODALITY.

Finally, in order to retrieve the number of articles, an aggregate function count is applied on the class QUERY.

\section{2) Percentage of abandoned sessions}

- The classes USER, PERFORMS-SESSION, SESSION, INTERACTION, QUERY and MODALITY are selected.

- Selection of queries with status Interrupted: The label of the edge $\langle S T A T U S, S T R I N G\rangle$ is set to Interrupted.

- The slice operator is applied on the temporal role-node PERFORMSSESSION with parameter all. 
- The interval time-display operator is applied on the role-node $r$ with parameter last-interval in order to retrieve the last interval of each session.

- The slice operator is applied on the temporal role-node INTERACTION with temporal predicate 'during $(i)$ '.

- The grouping time-display operator is applied on the role-node $r$ with parameter MODALITY and it is applied again on the new role-node $r$ with parameter $Q U E R Y$.

Finally, in order to retrieve the number of queries, an aggregate function count is applied to the class SESSION.

\subsection{Diagrammatic vs. Iconic Query Language}

The experiment reported in (Badre et al. 1996) compare two visual query languages: QBD* (Query by Diagram) (Angelaccio, Catarci \& Santucci 1990), adopting diagrams as visual formalism, and QBI (Query by Icon) (Massari \& Chrysanthis 1995), adopting icons as visual formalism. The experiment results support the hypothesis that the effectiveness of the two query language s varies depending on two independent variables, the user's skill and the query classes.

Hence, two variables were used in the experiment:

1. The user classes - the experiment classified the users into two groups: skilled and unskilled users;

2. The query classes - the queries were classified in terms of two parameters: the maximum semantic distance and the overall number of cycles in the query paths (a path is defined in (Badre et al. 1996) as a sequence of adjacent class-nodes and role-nodes in a Typed Graph, always starting and ending with a class-node). So, the experiment consider three query classes: close-uncyclic, with low-medium semantic distance and no cycles; closecyclic, with low-medium semantic distance and cycles; and far-uncyclic, with high semantic distance and no cycles (details about this classification are out of scope of this paper).

The evaluation measures used in the experiment are: completion time of the users'queries and accuracy.

From the representation of the user's stereotype in Figure 2, and given the two user classes adopted in the experiment, it results that the users'classification depends only on the features frequency of interaction and familiarity with the database content. So, the values of these features are $\mathrm{H}$ for a skilled user and $\mathrm{L}$ for an unskilled one. For the class $Q U E R Y$ in the Interaction GMDB, we consider a set of queries $\left\{Q_{1}, Q_{2}, \ldots, Q_{n}\right\}$ which are grouped into query classes close-uncyclic, close-cyclic, far-uncyclic. 
According to the experiment, each user performs a session $S_{i}$ with a fixed number of queries $\left\{Q_{1}, Q_{2}, \ldots, Q_{n}\right\}$, where each query $Q_{i}$ is expressed by two kinds of modalities, namely $M_{1}$ (representing the diagrammatic modality) and $M_{2}$ (representing the iconic modality). As we said above, a query gets a status at the end of the time interval of the last modality by which it is expressed. This happens in the general case of a multi-paradigmatic interface. Considering the specific case of this experiment, the user specifies a query through either the diagrammatic or the iconic modality, so there is no change of modality during the query specification. In this experiment, we do not consider intervals whose end instant is associated with an Interrupted status in the interpretation of the temporal role-node STATUS.

In (Badre et al. 1996), the experiment gave the following results (in this paper we do not consider the performance measure accuracy):

1. Average time to construct a query as a function of the skill level;

2. Average time to construct a query as a function of the query classes;

3 . Average time per query.

In order to obtain the result in each of the cases above, we apply the TVQOs on the Interaction $G M D B$ as follows. For all queries there are two common preliminary operations, namely:

- Selection of either diagrammatic (QBD*) or iconic (QBI) modality: The label of the edge $\langle I N T E R A C T I O N, M O D A L I T Y\rangle$ is changed into either $M_{1}$ or $M_{2}$.

- Selection of queries with status Completed: The label of the edge $\langle S T A T U S, S T R I N G\rangle$ is set to Completed.

\section{1) Average time to construct a query as a function of the skill level:}

- The classes USER, HAS-STEREOTYPE, STEREOTYPE, FEATURE, FREQUENCY, FAMILIARITY, PERFORMS-SESSION, SESSION, INTERACTION, MODALITY, QUERY, STATUS and STRING are selected.

- Selection of either skilled or unskilled users:

The label of the edges $\langle F E A T U R E, F A M I L I A R I T Y\rangle$ and $\langle F E A T U R E, F R E Q U E N C Y\rangle$ are changed either into $L$, in order to select the unskilled users or into $H$, for the skilled ones.

- Selection of recent skilled or unskilled users: snapshot on the temporal role-node HAS-STEREOTYPE with parameter end(now).

- Interactions occurring during the time of the experiment: slice on the temporal role-node INTERACTION with parameter during $(I)$ (I corresponds to the time period of the experiment).

- Intervals corresponding to each query expressed by either $\mathrm{QBD}^{*}$ or $\mathrm{QBI}$ : 
grouping time-display on the class $r$ resulting from the previous slice, collapsing the class MODALITY.

In order to retrieve the time needed to construct a query expressed by either $\mathrm{QBD}^{*}$ or QBI, the function duration( $\left.i\right)$ is applied to the class $i$ resulting from the grouping time-display. Next, an aggregate function avg is applied on the result of the function duration $(i)$, that is, avg(duration $(i))$.

2) Average time to construct a query as a function of query classes:

- The classes USER, PERFORMS-SESSION, SESSION, INTERACTION, MODALITY, QUERY, IS-ELEMENT, QUERY-CLASS, STATUS and STRING are selected.

- Selection of close-uncyclic, close-cyclic and far-uncyclic queries: The label of the edge $\langle I S-E L E M E N T, Q U E R Y-C L A S S\rangle$ is changed into either close-uncyclic, or close-cyclic, or far-cyclic.

All operations described for case (1) above are applied also in this case, except those described in items $1,2,3$.

\subsection{Discussion}

The two usability experiments 'we have previously described are comparative studies among the different ways to access information, in order to explore the effectiveness of an interaction modality. The first experiment comproves the hypothesis that the hypertext modality would perform better than the index accesses modality, while the second experiment comproves the hypothesis that the effectiveness of an diagrammatic or an iconic query language varies, depending on the class of queries and kind of users.

As we reported in the introduction, some other experiments have been conducted aiming at demonstrating the same hypothesis of the last experiment. However, very few empirical studies aiming at testing and validating the effectiveness of various query styles and interfaces have been conducted in the database field (Badre et al. 1996). One of the main reasons is the intrinsically complex and time-consuming nature of the process of conducting experiment with real users. Indeed, this process involves several activities, such as setting up the experiment environment, selecting the users, planning the tasks, measuring the performance, analyzing the results, etc.

Within this context, the idea of providing a framework which can be incorporated within any VQS is an useful approach for detecting the early failures/errors that may occur, even during the system design. The choice of the main framework components is based on the existing literature on VQS usability experiments. All such experiments consider among the essential vari- 
ables the user's skill, the query classes, the interaction modalities and access methods within specific tasks.

It is worth noting that the availability of temporal data allows a deeper analysis of the system usability. In particular, it is fundamental to keep track of the evolution of the user's interaction, and notice how this evolution affects the system ease-of-use. Finally, temporal measures well characterize the users'performances.

\section{CONCLUSIONS}

In this paper we have presented a framework for modeling the temporal data about the user-system interaction resulting from usability experiments. Such a framework is integrated within a VQS for querying temporal databases. The overall system is based on a model-independent formalism, equipped with basic visual operators.

Through the TVQOs applied on the temporal information generated during the user-system interaction, several temporal queries can be specified such as: What is the average response time for each query?, What was the average time to complete a query for each modality?, Since when is John Smith a repetitive user? or What is the history of the users'sessions during 1997?.

The availability of a homogeneous access to the application data and interaction histories makes it possible a dynamic evaluation of the system usability, since all time-related information generated during a real interaction between the user and the database can be analyzed at any time. Moreover, our approach avoids the development of experiments that are performed most of the time in artificial environments with artificial tasks and removing the user from her/his normal work situation. Data about the user's interaction may be produced during the standard activity and then analyzed, avoiding the time-stress effect. Time-stress is defined as an externally induced urgency to complete an assigned task within a specified or limited amount of time, which is known to have an adverse effect on human performance (Wallace, Anderson \& Schneiderman 1987).

In short, the main contribution of our approach is the integration of the temporal data about the user model and the user's interaction inside the query environment, so to enforce the link between the user interaction and modeling and data modeling and querying. A further benefit deriving from our approach is the consistent and well controlled evaluation of the VQS usability.

The overall system is actually under implementation, and the next step will be to carry out complete usability tests in real settings. Moreover, the future work will aim to extend the expressive power of the visual temporal operators. 


\section{REFERENCES}

Allen, J. (1983), 'Maintaining knowledge about temporal interval', Communications of ACM 26(1), 832-843.

Angelaccio, M., Catarci, T. \& Santucci, G. (1990), 'QBD*: A graphical query language with recursion', IEEE Transaction on Software Engineering 16(10), 1150-1163.

Badre, A., Catarci, T. \& Santucci, G. (1996), Comparative ease of use of a diagrammatic versus an iconic query language, in K. J., ed., 'Third Int. Workshop on User Interfaces to Database Systems, Edinburgh'.

Bell, J. (1993), The experiences of new users of a natural language interface to a relational database in a controlled setting, in C. R., ed., 'Interface to Database Systems Glasgow 1992', Workshop in Computing, SpringerVerlag, London, pp. 433-454.

Catarci et al., T. (1996), 'A graph-based framework for multiparadigmatic visual access to databases', IEEE Transactions Knowledge on Data Engineering 8(3), 455-475.

Catarci et al., T. (1997), 'Visual query systems: Analysis and comparison', Journal of Visual Languages and Computing (8), 215-260.

Catarci, T., Santucci, G. \& Angelaccio, M. (1993), 'Fundamental graphical primitives for visual query languages', Information Systems 18(2), 7598.

Catarci, T., Santucci, G. \& Cardiff, J. (1997), 'Graphical interaction with heterogeneus databases', VLDB Journal 6(2), 97-120.

Chang, S., Costabile, M. \& Levialdi, S. (1993), A framework for intelligent visual interface design for database systems, in C. R., ed., 'Interface to Database Systems, Glasgow 1992', Workshop in Computing, SpringerVerlag, London, pp. 377-391.

Fernandes Silva, S. \& Catarci, T. (1998), 'Homogeneous access to temporal data and interaction histories in adaptive interfaces for databases', submitted paper.

Fernandes Silva, S., Schiel, U. \& Catarci, T. (1997), Visual query operators for temporal databases, in M. R. \& K. L., eds, '4th Workshop on Temporal Representation and Reasoning, Daytona Beach Florida', IEEE Computer Society, pp. 46-54.

Hull, R. \& King, R. (1987), 'Semantic database modeling: Survey, applications and research issues', ACM Computing Surveys 19(3), 201-260.

Jensen et al., C. (1994), 'A consensus glossary of temporal database concepts', SIGMOD RECORD 23(1).

Kass, R. \& Finin, T. (1989), 'The role of user models in cooperactive interactive systems', International Journal of Intelligent Systems 4, 81-112.

Kim, W. (1989), A model of queries for object-oriented databases, in 'Proc. 15th Int. Conf. on Very Large Databases, Amsterdam'.

Leventhal et al., L. (1995), Searching without a keyboard in a multimedia 
environment, in N. K. et al., ed., 'INTERACT 95', Chapmen \& Hall: Oxford, pp. 241-246.

Massari, A. \& Chrysanthis, P. (1995), Visual query of encapsulated objects, in 'Proc. of the 5th Int. Workshop on Research Issues on Data Engineering, Taipei, Taiwan', pp. 18-25.

Morris, R. \& Khatib, L. (1997), Entities and relations for historical relational databases, in R. Morris \& L. Khatib, eds, '4th Workshop on Temporal Representation and Reasoning, Daytona Beach, Florida', IEEE Computer Society, pp. 180-186.

Paton et al., N. (1995), Techniques for the effective evaluation of database interfaces, in C. R., ed., 'Interfaces to Database System, Lancaster 1994', Workshop in Computing, Springer-Verlag, London, pp. 306-328.

Resneir, P. (1988), Query Languages. Handbook of Human-Computer Interaction, North-Holland.

Shneiderman, B. (1994), 'Dynamic queries for visual information seeking', IEEE Software 11, 70-77.

Shneiderman et al., B. (1989), 'Evaluating three museum installations of a hypertext system', Journal of the American Society for Information Science 40(3), 172-182.

Wallace, D., Anderson, N. \& Schneiderman, B. (1987), Time stress effects on two menu selection systems, in 'Proc. 31st Annual Meeting - Human Factors Society', pp. 727-731.

\section{BIOGRAPHY}

Sônia Fernandes Silva received her MS degree in computer science from the Federal University of Paraíba, Brazil, in 1995. She is a PhD student in computer science at Electric Engineering Department of the Federal University of Paraíba and she has been a visiting student at the University of Rome.

Her main research interests includes visual formalisms for representation and querying of temporal databases, visual languages for historical databases, visual temporal operators and visualization of temporal information.

Tiziana Catarci received her $\mathrm{PhD}$ in computer science from the University of Rome, where she is currently an Assistant Professor.

Her main research interests are in theoretical and application oriented aspects of visual formalisms for databases and database design, areas on which she has published over 70 papers and 5 books. Dr. Catarci is regularly in the programming committees of the main database conferences and is in the steering committee of the Working Conference on Advanced Visual Interfaces (AVI). She has been guest co-editor of two recent special issues of the JVLC (on "Visual Query Languages") and of the ACM SIGMOD Record (on "Information Visualization"). 